\title{
UNA OBRA INÉDITA DEL ESCULTOR ANTONIO SUSILLO: EL SUEÑO DE UN ÁNGEL (1885)
}

\author{
AN UNKNOW WORK BY THE SCULPTOR \\ ANTONIO SUSILLO: THE DREAM OF AN ANGEL (1885)
}

\author{
Alicia Iglesias Cumplido \\ Grupo de investigación HUM948: Imagenan. Arts \& Media. Imagen Artística de \\ Andalucía. España \\ aiglesiascumplido@hotmail.com
}

\begin{abstract}
El artículo presenta una escultura inédita de Antonio Susillo, firmada y fechada en el año 1885, en fecha inmediata a su estancia en París y previa a la posterior en Roma. Dada la temprana fecha, la obra supone una aportación importante para la identificación de los rasgos morfológicos que determinaron la evolución del escultor.

Palabras clave: Antonio Susillo; escultura; Realismo; París, Roma.
\end{abstract}

This paper shows a new sculpture by Antonio Susillo, signed and dated in 1885, immediately after Paris and before Rome's travel. This sculpture is an important contribution to identify morphological features of Antonio Susillo.

Keywords: Antonio Susillo; sculpture; Realism; Paris; Rome.

El pequeño grupo escultórico El sueño de un Ángel", firmado "A. Susillo, 85", fue un regalo personal del escultor al pintor Antonio Cavallini Casellini ${ }^{2}$, con el que trató en sus trabajos para el palacio de San Telmo por encargo del duque de Montpensier. En la base del grupo aparece incisa la frase "sin concluir".

${ }^{1}$ Colección particular, barro, $33 \times 25 \times 25 \mathrm{~cm}$.

2 Bissone, 1836-Marmolejo, 1905. 
Si tenemos en cuenta el perfil biográfico de Antonio Susillo ${ }^{3}$, actualizado por Joaquín Álvarez $\mathrm{Cruz}^{4}$, podremos calibrar mejor la importancia de la identificación de esta escultura inédita, que permite estudiar con mayor información los rasgos morfológicos del escultor en fecha relativamente temprana y en un momento previo al modelado de la mayor parte de su obra conocida e identificada ${ }^{5}$. Por eso es prioritario el análisis morfológico de este pequeño grupo escultórico.

La obra está compuesta por dos figuras, algo muy frecuente en el repertorio del escultor: el retrato infantil como protagonista del asunto y el ángel protector cuyo significado alegórico fue muy frecuente en su obra, tanto como la asociación directa de dos figuras, una terrenal y otra sobrenatural, con las que solía captar un instante propio de un relato literario.

Es frecuente que en esa relación de dos figuras, Antonio Susillo situase una sentada, siempre el protagonista terrenal y a su lado aparezca en pie la alegórica que lo protege, amenaza o anuncia, según el tema de la representación. Esa composición ya la había planteado de modo similar en Escena de Fausto ${ }^{6}$, fechada en 1870-1879, un bajorrelieve en el que destaca una fuga en perspectiva que marca las diferencias. Después la recordó en Un ángel más ${ }^{7}$, en 1892, que difiere porque el ángel aparece en vuelo y porta en sus brazos a un niño que señala con su mano derecha al cielo. Dicho esquema aún tuvo similitudes en el grupo principal del Monumento a Cristóbal Colón en Valladolid, en 1892-1894, en el que aparece el almirante arrodillado y justo detrás en vertical una figura alegórica que lo anuncia y lo protege. Como puede verse, el esquema es parecido en obras con formatos muy distintos y variantes no menos significativas.

Es un niño de corta edad, dormido tumbado boca arriba, vestido conforme a la moda infantil de las clases sociales altas, sobre un sillón de tipo inglés con tapizado capitoné. Las piernas sobresalen del sillón y la cabeza con la boca levemente

${ }^{3}$ SEDANO, Eugenio: Estudio de estudios. Artículos-siluetas de pintores y escultores sevillanos. Sevilla, 1896, pp. 44-50; CASCALES MUÑOZ, José: Las Bellas Artes plásticas. La pintura, la escultura y cerámica artísticas desde el siglo XIII hasta nuestros días. Vol. II. Toledo, 1929, p. 44; LÓPEZ DEL TORO, José: "Cinco cartas del escultor Susillo al marqués de Pickman", Archivo Hispalense, 15, 1946, pp. 77-113; y DE LA BANDA Y VARGAS, Antonio: "Antonio Susillo. Académico de Bellas Artes", Temas de estética y arte, 11, 1997, pp. 69-74.

4 ÁLVAREZ CRUZ, Joaquín: "Notas biográficas sobre el pintor Antonio Susilllo", Laboratorio de Arte, 10, 1997, pp. 523-525.

5 ILLANES, Antonio: "Antonio Susillo y su ingente obra", Boletín de Bellas Artes, 3, 1975, pp. 11-26; DE LA BANDA Y VARGAS, Antonio: "Datos para la historia de la escultura sevillana del siglo XIX”, Boletín de Bellas Artes, 9, 1981, pp. 183-218; y BLÁZQUEZ SÁNCHEZ, Fausto: "Antonio Susillo", en La escultura sevillana en la época de la Exposición Iberoamericana (1900-1930). Ávila, 1989, pp. 112-113.

${ }^{6}$ Colección particular, barro, $16,1 \times 7,9 \mathrm{~cm}$.

${ }^{7}$ Colección particular, barro, 25,6 x 13,4 cm. 
abierta es muy expresiva. Los brazos están modelados de un modo muy suelto y el derecho presenta pérdidas de materia. El volumen de la cabeza destaca por un modelado muy preciso sobre las texturas sueltas y rugosas del vestido, en el que destacan las transparencias de las medias (Figura 1).

El ángel está sentado sobre uno de los brazos y el respaldo del sillón, con las grandes alas abiertas para mantener la estabilidad y la mirada y la expresión dirigida al niño. Igual que la figura infantil, destaca la volumetría de la cabeza y el modelado preciso y fino de la cara, que contrastan con la soltura de la túnica y con las texturas de las grandes alas. La relación con la figura infantil, dormida y por lo tanto desprotegida, lo presenta como un ángel de la guarda.

En conjunto, las dos figuras son muy dinámicas, la infantil por la caída relajada de su cuerpo, representando un sueño plácido bajo la protección del ángel. $\mathrm{Su}$ anatomía refleja seguridad, como si estuviese bajo la protección de su madre, con una caída moderada de la musculatura, pero muy expresiva y de fácil percepción. El ángel completa la composición desplazando una importante masa volumétrica a la parte trasera; sin embargo, la forma y el equilibrio que mantiene compensa los pesos y genera un centro de gravedad que le proporciona estabilidad y hace que se cree una línea directa entre la mirada del ángel y la cara del niño (Figura 2)

Las zonas intermedias, como la parte baja y trasera del sillón, presentan texturas rugosas, muy sueltas e informales, que coinciden con los juegos de luces impresionistas, y destacan frente a los detalles preciosistas del vestido del niño. De esta forma, las partes realistas, muy bien definidas, destacan sobre las superficies de barro movidas en las que los motivos se diluyen llegando a la abstracción. Todo esto confirma la evolución de Antonio Susillo, que asumió el realismo derivado del escultor Jean-Baptiste Carpeaux y las últimas novedades de aquel momento en la escultura francesa e introdujo en España el estilo internacional del que también partió Mariano Benlliure en sus primeras esculturas. En esas partes supo mantener las propiedades volumétricas del bulto redondo, como también lo hizo en los retratos y los detalles fundamentales de los relieves, a la vez que introdujo un modelado preciosista en lo accesorio, con una fuerte incidencia de la luz y contrastes de texturas acusados. Esto puede identificarse con la tendencia preciosista representada en pintura por Mariano Fortuny, en la que habría que encuadrar el grupo El sueño de un árabe ${ }^{8}$, del año 1890, en el que destaca el acabado pulimentado de la anatomía femenina sobre una base muy rugosa; y especialmente los relieves como El suspiro del moro 9 , del año 1891.

Como ya vimos, la figura infantil tuvo una destacada presencia en el repertorio de Antonio Susillo. Si lo comparamos con los niños de La primera contienda, del año 1882, la primera impresión es la de una representación con características

${ }^{8}$ Colección particular, barro, $140 \mathrm{~cm}$ de altura.

${ }^{9}$ Colección particular, barro, 56 x 18 x 42,5 cm. 
opuestas, ya que mientras aquellos son pura vitalidad subiendo sobre el cuerpo de su madre, este se presenta relajado y en postura distendida; sin embargo, las dos opciones responden a la dinámica del cuerpo en acciones diferentes, a lo que respondió con un mismo criterio naturalista, superado con la deformación de lo accesorio al proceder con un modelado suelto y a veces pictórico, más intenso con las simplificaciones lineales con el palillo. Los tipos físicos coinciden y lo mismo podemos comprobar en la vitalidad de los movimientos, en puntos extremos de la acción que representan. El tipo físico también coincide con el Niño Jesús del Descanso en la huida a Egipto y la Epifanía, en estos casos erguido y solemne como responde a una representación divina.

Una vez identificado un tipo infantil, hay que reconocer que todos los niños de Antonio Susillo son muy distintos entre sí, probablemente porque estudiase los modelos del natural, a excepción de la representación del Niño Jesús, ya que siendo un mismo personaje decidió representarlo siempre del mismo modo, con las mismas características físicas. También es muy significativo que los cambios de escala no suponen un problema para mantener las características de su estilo. Las diferencias son mayores con las figuras juveniles, ya que estos personajes, al tener más edad, suelen adoptar posturas complejas e infrecuentes en los niños. Como curiosidad, uno de esos jóvenes, el que aparece en El sueño del Quijote, está en una actitud contemplativa que coincide con la relajación del niño dormido en esta obra.

El ángel también estuvo muy presente en la escultura de Antonio Susillo, como quedó reseñado en una serie de obras bien identificadas y estudiadas, y en numerosos ejemplos de pequeños grupos y relieves conocidos por una colección de antiguas fotografías en albúmina tomadas por Eugenio Gómez a finales del siglo $\mathrm{XIX}^{10}$, la mayoría pendientes de estudio. La iconografía ya está muy definida en Un alma infantil, hacia 1880, obra en la que aparece un modelo físico con un género indefinido, con rasgos suaves muy bien resueltos en su ambigüedad, y grandes alas abiertas representadas en distintos niveles para aportar movimiento y equilibrio cuando es necesario. Esas alas, muy significativas desde el punto de vista plástico, están modeladas de un modo muy suelto y a veces buscando el dibujo con incisiones sobre la superficie (Figura 3).

Mientras en Ángel Apocalíptico ${ }^{11}$, de 1890, optó por representarlo sentado y en un momento de reposo previo a la acción final, este otro es un ángel de la guarda que se presenta en equilibrio y permanente alerta para defender la

${ }^{10}$ Fotógrafo establecido en San Juan de la Palma, $\mathrm{n}^{\mathrm{o}}$ 12, Sevilla.

11 GONZÁLEZ GÓMEZ, Juan Miguel y ROJAS-MARCOS GONZÁLEZ, Jesús: "Cuatro nuevas obras del escultor Antonio Susillo", Laboratorio de Arte, 23, 2011, pp. 391-414; y GONZÁLEZ GÓMEZ, Juan Miguel y ROJAS-MARCOS GONZÁLEZ, Jesús: "La obra de Antonio Susillo en la Casa-Palacio de la Condesa de Lebrija", Temas de estética y arte, 27, 2013, pp. 177-219. 
inocencia del niño dormido. La grandeza de las alas abiertas lo relacionan con las representaciones en las que lo equiparó con las victorias clásicas, pongamos por caso la que remata el proyecto de Monumento a la infanta María Luisa, de 1893. También hay que tener en cuenta que el interés por el barro y las texturas lo relacionan con el ángel de El sueño de la novicia, hacia 1890, con la diferencia muy destacada del distinto criterio de ejecución entre uno y otro, ya que el que aquí presentamos está modelado con un gran agilidad y desplazamientos de la materia, y el de este relieve se diluye tanto que llega a estar prácticamente dibujado sobre el barro fresco en una eficaz técnica mixta.

El grupo escultórico El sueño de un ángel es un original de Antonio Susillo hasta ahora inédito, avalado por la firma en la base y por su localización en la colección de los herederos del pintor Antonio Cavallini Casellini, amigo personal del escultor. En esa firma aporta la cifra " 85 ", con lo que también queda identificado el año de producción (Figura 4). Por lo tanto, es anterior a la mayoría de las esculturas monumentales conocidas de Antonio Susillo, en una fecha inmediata a su estancia en París y previa a su estancia en Roma, y en el inicio de su madurez artística. En ese momento, las características analizadas lo presentan como un artista muy moderno y abierto a las características de la escultura realista difundida desde París, cuya proyección internacional lo convirtió en el renovador de la escultura sevillana de su época.

La obra indica también la gran personalidad artística de Antonio Susillo, que en ese momento ya mostró un estilo personal que lo distinguió de Carpeaux y otros escultores franceses, y no tiene comparación con otros escultores españoles y mucho menos sevillanos en esa fecha. Lo hace con dos conceptos: uno, el contenido literario que lo relaciona con los escritores postrománticos de su época, incluido Gustavo Adolfo Bécquer; y el otro, de carácter formal, con una habilidad de modelado que lo situó en la modernidad de su tiempo.

Puede decirse que el ángel es una de las representaciones más frecuentes en el repertorio alegórico de Antonio Susillo; aparece en muchos de sus relieves, y como ya se dijo, puede hacerlo con distintos significados simbólicos. La iconografía ya está muy definida en Un alma infantil ${ }^{12}$, fechada hacia 1880 , en el que aparece modelado muy abocetado y con las alas bien abiertas. En la Alegoría del inicio del reinado de Isabel $I I^{13}$, en 1876-1882, se muestra como un ángel triunfante equiparable a una Victoria. La relación con el simbolismo parisino lo caracteriza en el Descanso en la huida a Egipto ${ }^{14}$, en 1882, en el que está sentado sobre un busto colosal que sirve de pantalla a la Sagrada Familia, sentado abrazándose

${ }^{12}$ Colección particular, barro, 33 x 18 cm. GONZÁLEZ GÓMEZ, Juan Miguel y ROJAS-MARCOS, Jesús: "Cuatro nuevas obras...", op. cit., p. 409.

13 Ibidem, p. 410.

${ }^{14}$ Casa del Niño Jesús, Málaga, barro, 85 cm. GONZÁLEZ GÓMEZ, Juan Miguel y ROJAS-MARCOS, Jesús: “Cuatro nuevas obras...”, op. cit., p. 411. 
a una rodilla, con las alas abiertas y a distinta altura para compensar la inestabilidad. Uno de los más representativos es el protagonista de Ángel Apocalíptico ${ }^{15}$, en 1890, representado sentado y acodado sobre un libro. La colocación de las piernas a distintos niveles y el movimiento helicoidal del cuerpo se corresponden con las alas abiertas para mantener la estabilidad. El delicado movimiento de los palillos prácticamente dibuja sobre el barro parte de las alas del ángel de $E l$ sueño de la novicia ${ }^{16}$, de fecha parecida. La verticalidad y el impacto de las grandes alas abiertas hubiesen caracterizado al que habría de rematar el Monumento a la Infanta María Luisa ${ }^{17}$, como puede verse en el proyecto del mismo dibujado por el escultor, del año 1893. Todos presentan grandes alas abiertas, aunque puede variar la posición.

Las representaciones infantiles y juveniles son menos frecuentes en la obra de Antonio Susillo que las de ángeles y figuras alegóricas; sin embargo, aparecen en su repertorio con la frecuencia suficiente como para establecer las pautas de representación que caracterizaron al escultor. Las referencias naturalistas y el intenso dinamismo de los niños que luchan sobre la anatomía desnuda de su madre en el grupo monumental La primera contienda ${ }^{18}$, del año 1885, tienen el nivel artístico suficiente para proponerlos como prototipo de la estética realista de la que hablamos. Antonio Susillo le dio una gran importancia al estudio de la anatomía, y aunque introdujo efectos pictóricos que dinamizan las superficies mantuvo el volumen propio de la escultura. También le dio prioridad a los efectos expresivos relacionados con los movimientos. Con esto concuerda el niño de $E l$ sueño de un ángel.

En este también se identifican el volumen y el natural de un modo similar al niño del relieve del Descanso en la huida a Egipto, del año 1882, aunque sea una parte pequeña del mismo y haya que distinguirlo entre la profusión preciosista. Lo comentado y la colocación diagonal y el dinamismo lo relacionan con los niños de La primera contienda. Prácticamente lo repitió en el relieve de La Epifanía ${ }^{19}$, que habría que fechar en la misma época, posible boceto muy deteriorado,

${ }^{15}$ Colección Mariano Bellver, barro, 54 x 44 x $21 \mathrm{~cm}$. ROJAS-MARCOS GONZÁLEZ, Jesús: "Tres obras inéditas de Susillo. Un ángel y dos relieves pasionistas", Laboratorio de Arte, 26, 2014, p. 297. Pudiera haber una segunda versión en colección particular, barro, $20,9 \times 16,9 \mathrm{~cm}$.

${ }^{16}$ Procedente de la colección Lacavé de Aspe.

${ }^{17}$ LAFITA, Teresa: Sevilla turística y cultural. Fuentes y monumentos públicos. Madrid-Sevilla, 1998, p. 64.

18 Boceto en la colección del marqués de Aracena, restaurado por Fernando Manuel del Toro. Original del grupo en el Museo de Bellas Artes de Sevilla.

${ }^{19}$ Colección particular, barro, 23 × 37 x 2,5 cm. Restaurado por Manuel Chiappi en Restauraciones Buendía. Reconstruyó la zona a la espalda de la Virgen, la figura de San José y partes del arco y el techo. 
si bien ninguno de esos niños aparece ni tumbado ni dormido como el que aquí presentamos.

En cuanto a la inestabilidad de la representación de este niño, habría que tener en cuenta la postura del joven que comparte montura sobre un camello en Escena oriental, inclinado hacia detrás, pero sin llegar a estar tumbado; también la del joven del grupo alegórico La Náutica, del Monumento a Cristóbal Colón en Valladolid, del año 1893, que se echa sobre la figura femenina y le da la espalda al espectador, como será pronto frecuente en el Modernismo; e incluso la del joven que ocupa un lugar clave en el relieve El sueño del Quijote ${ }^{20}$, entre la figura ecuestre del personaje de Cervantes y la itinerante de Sancho, como testigo del diálogo literario.

Fecha de recepción: 30 de octubre de 2018

Fecha de aceptación: 1 de julio de 2019

${ }^{20}$ Colección particular, barro, 12,2 x 15,4 cm. 


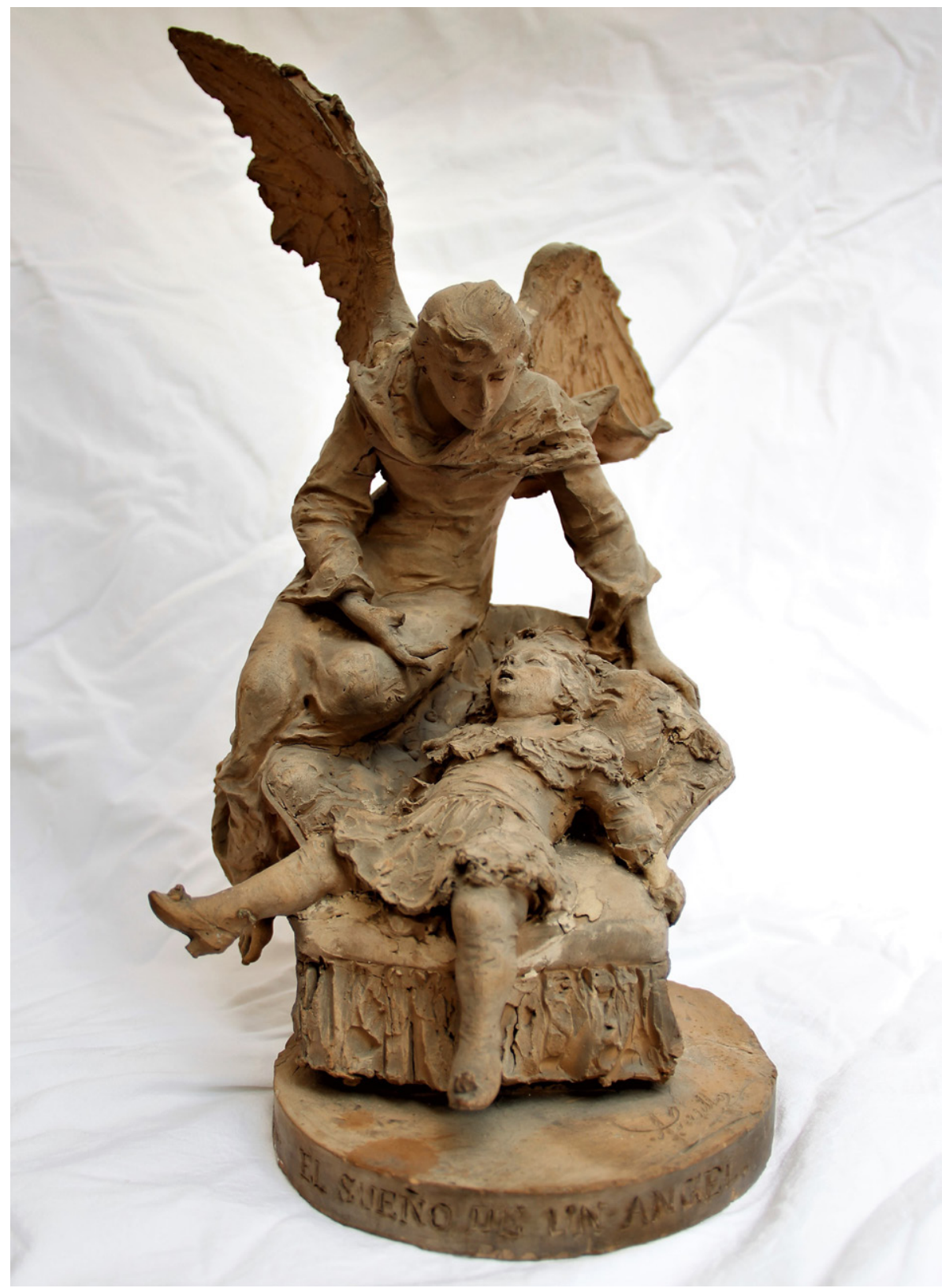

Figura 1. Antonio Susillo, El sueño de un Ángel, 1885. 


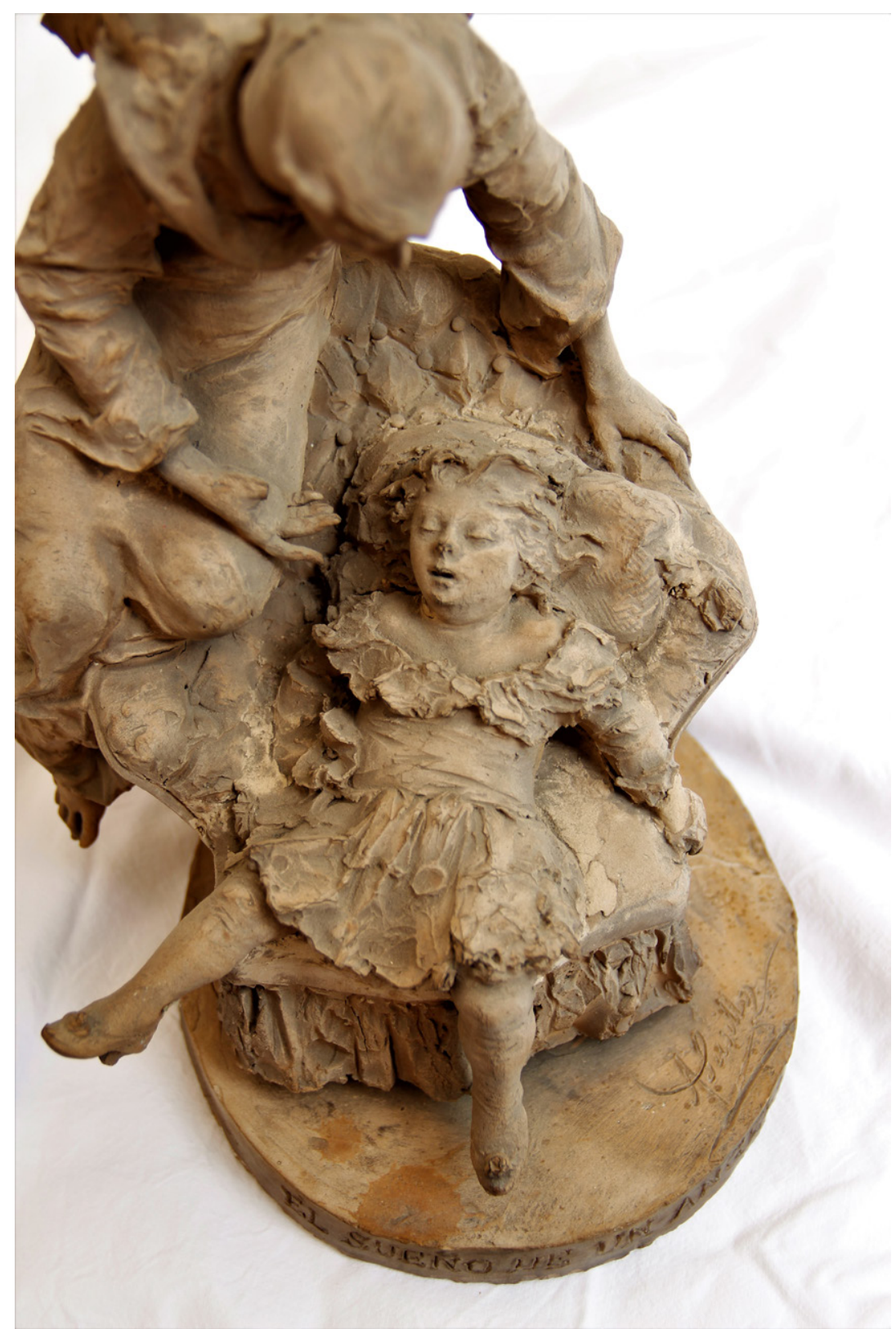

Figura 2. Antonio Susillo, El sueño de un Ángel (detalle del niño), 1885. 


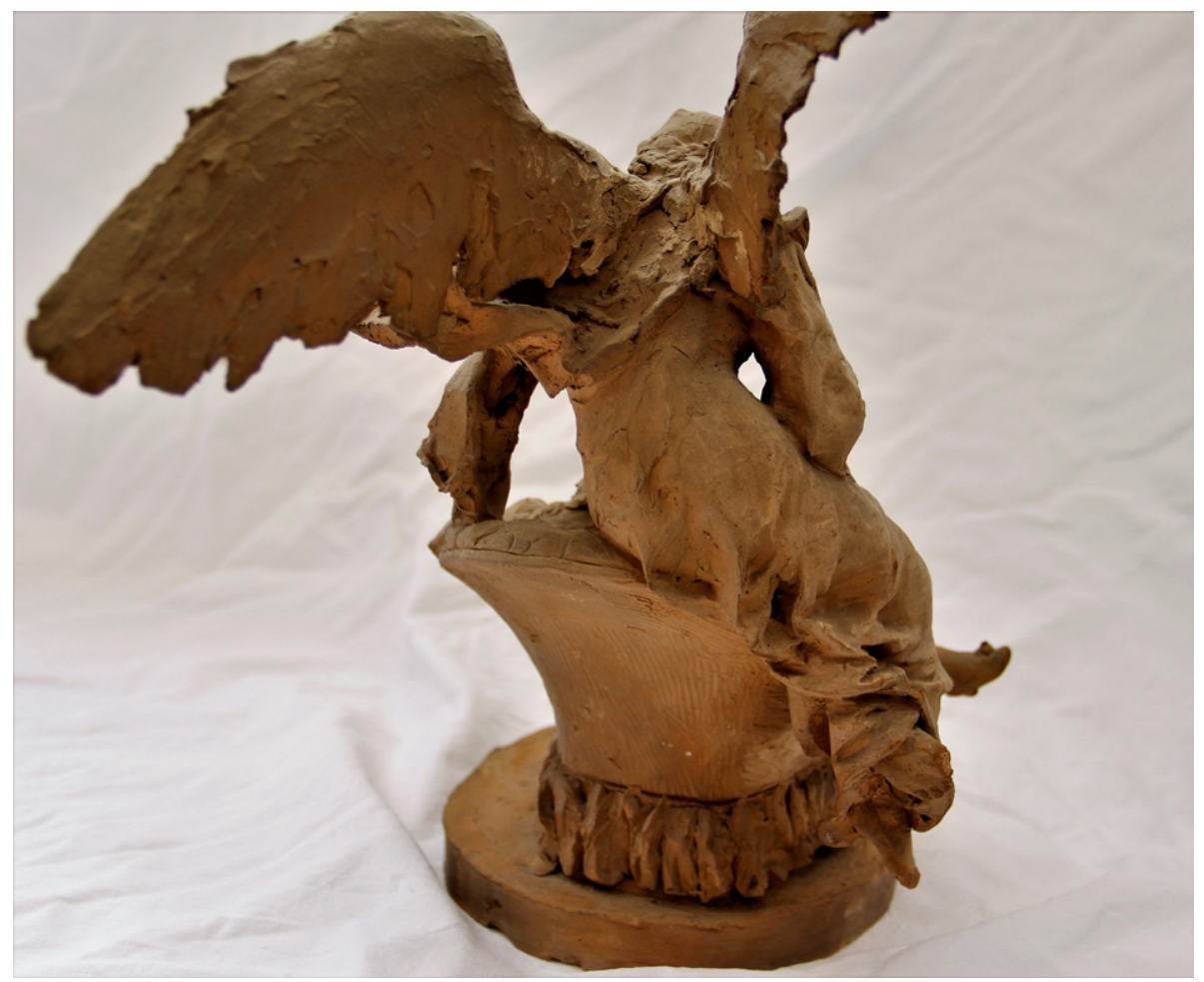

Figura 3. Antonio Susillo, El sueño de un Ángel (vista trasera), 1885.

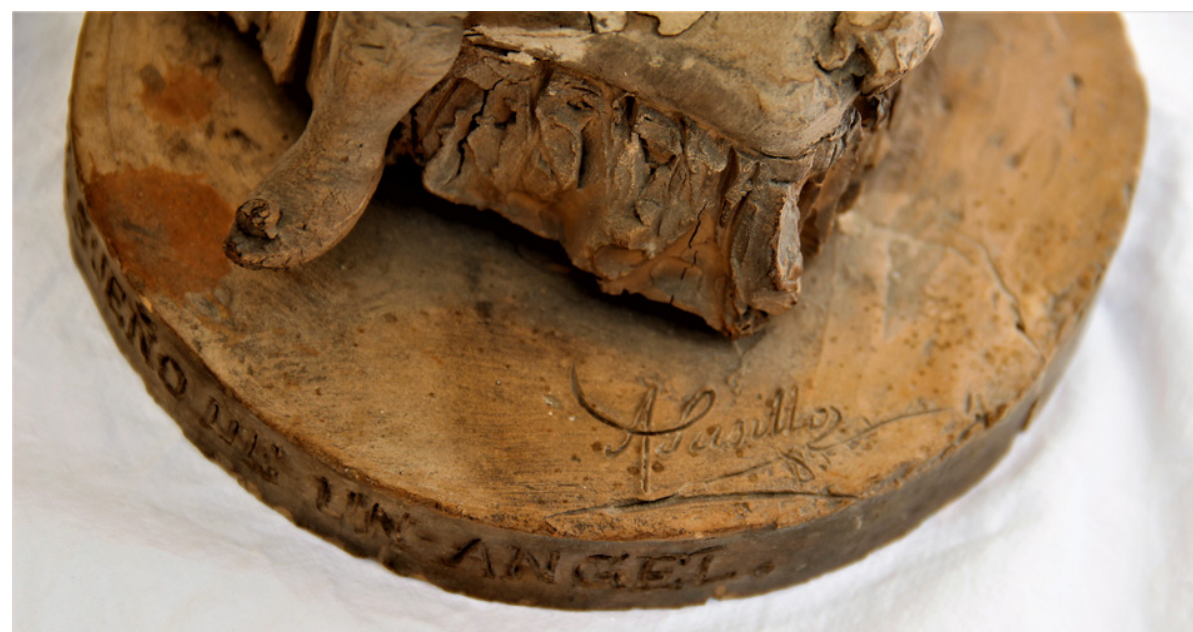

Figura 4. Antonio Susillo, El sueño de un Ángel (firma del escultor), 1885.

LABORATORIO DE ARTE 31 (2019), pp. 693-702, ISSN 1130-5762

e-ISSN 2253-8305 - DOI http://dx.doi.org/10.12795/LA.2019.i31.43 